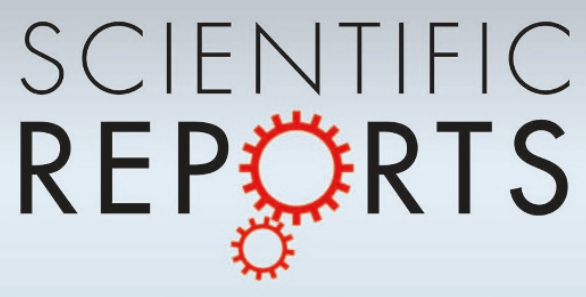

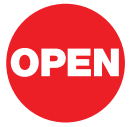

SUBJECT AREAS:

CANCER IMAGING

MAGNETIC RESONANCE

IMAGING

NANOPARTICLES

OPTICAL IMAGING

Received

13 November 2012

Accepted

15 March 2013

Published

8 April 2013

Correspondence and requests for materials should be addressed to C.J. (jiangchen@shmu. edu.cn)

\section{A choline derivate-modified nanoprobe for glioma diagnosis using MRI}

\author{
Jianfeng Li', Shixian Huang' ', Kun Shao', Yang Liu', Sai An', Yuyang Kuang', Yubo Guo', Haojun Ma', \\ Xuxia Wang ${ }^{2} \&$ Chen Jiang'
}

\begin{abstract}
'Department of Pharmaceutics, School of Pharmacy, Fudan University, 826 Zhangheng Road, Shanghai 201203, China, ${ }^{2}$ State Key Laboratory of Magnetic Resonance and Atomic and Molecular Physics, Wuhan Institute of Physics \& Mathematics, Chinese Academy of Sciences, Wuhan 430071, Hubei, China.
\end{abstract}

Gadolinium (Gd) chelate contrast-enhanced magnetic resonance imaging (MRI) is a preferred method of glioma detection and preoperative localisation because it offers high spatial resolution and non-invasive deep tissue penetration. Gd-based contrast agents, such as Gd-diethyltriaminepentaacetic acid (DTPA-Gd, Magnevist), are widely used clinically for tumor diagnosis. However, the Gd-based MRI approach is limited for patients with glioma who have an uncompromised blood-brain barrier (BBB). Moreover, the rapid renal clearance and non-specificity of such contrast agents further hinders their prevalence. We present a choline derivate $(\mathrm{CD})$-modified nanoprobe with $\mathrm{BBB}$ permeability, glioma specificity and a long blood half-life. Specific accumulation of the nanoprobe in gliomas and subsequent MRI contrast enhancement are demonstrated in vitro in U87 MG cells and in vivo in a xenograft nude model. BBB and glioma dual targeting by this nanoprobe may facilitate precise detection of gliomas with an uncompromised BBB and may offer better preoperative and intraoperative tumor localization.

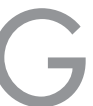

liomas are currently the most common type of primary central nervous system (CNS) cancer in adults and are the most lethal in children ${ }^{1}$. Despite recent advances in brain cancer therapy, the prognosis remains poor for patients diagnosed with malignant gliomas. For example, glioblastoma patients have a median survival time of less than 15 months after surgical intervention followed by radiotherapy and chemotherapy ${ }^{2}$. The effectiveness of surgical resection is primarily limited by a poor preoperative diagnosis and intraoperative visual contrast between the tumor and normal brain tissue because of the infiltrative nature of these tumors. Thus, the diagnosis of gliomas and the more precise delineation of tumour margins are imperative for improving surgical outcomes.

Currently, Gd chelate contrast-enhanced MRI is a preferred choice for preoperative tumor localization ${ }^{3}$. Gd chelates, such as DTPA-Gd, have been approved for clinical use in $\mathrm{T}_{1}$-weighted MRI. These small molecular contrast agents diffuse into the extracellular interstitial compartment of tumor, where BBB is disrupted, and lead to MR signal enhancement. However, approximately $30 \%$ of anaplastic astrocytomas do not show any MR signal enhancement due to the uncompromised $\mathrm{BBB}^{4}$. Moreover, the rapid renal clearance and non-specificity of such contrast agents further hinder their prevalence ${ }^{5}$. Therefore, probes with BBB permeability, long blood half-lives and high specificity are needed.

Dendrimer-based nanoprobes are ideal diagnostic agents for medical imaging applications, with advantages that include prolonged blood half-lives and enhanced permeability and retention (EPR) effect. A receptormediated endocytosis mechanism can produce tumor-specific nanoprobe internalization ${ }^{6-8}$. However, this active targeting requires accumulation in the extracellular interstitial compartment of tumor, which depends on the EPR effect. Thus, nanoprobes are insufficient to diagnose gliomas with uncompromised BBB. Herein, a new strategy based on the metabolism characteristics of tumors was used to integrate BBB permeability and tumor specificity into nanoprobes. Because they are highly malignant, glioma cells have an extreme requirement for choline as an extrinsic substrate to synthesise membrane phospholipids via the Kennedy pathway ${ }^{9}$. Overexpression of choline transporters (ChT), including choline transporter-like (CTL1) proteins, in glioma cells contributes to the high uptake of choline and choline derivates ${ }^{10,11}$. BBB ChT are responsible for choline uptake in the brain to support the neurological requirements of a normal brain ${ }^{12}$. Thus, choline transporters could facilitate BBB permeation by choline or choline derivatives, which would accumulate in glioma cells. Fluorine-18 $\left({ }^{18} \mathrm{~F}\right)$-labelled choline has been used to image a recurrent anaplastic astrocytoma with a high tumor-to-cortex ratio in a patient, which 
indicates that choline uptake in a normal brain did not decrease the signal-to-noise ratio for glioma imaging ${ }^{13}$. In our previous study, a novel CD with high BBB ChT affinity successfully facilitated gene delivery into the $\mathrm{CNS}^{14}$. A CD with even higher affinity than choline chloride was used as a novel choline transporter ligand that may target both BBB ChT and glioma ChT. The "proof of concept" is as follows: nanoprobes tagged with this ligand can penetrate the $\mathrm{BBB}$ and target gliomas. Such nanoprobes may detect gliomas more accurately and allow better preoperative and intraoperative tumour imaging.

\section{Results}

Nanoprobe design, synthesis and targeting strategies. Dendrigraft poly-L-lysines (DGL) are cationic, biodegradable, monodispersed, well-defined, and have the main properties of dendrimers. Thirdgeneration (G3) DGL have 123 primary amines, which indicates a high loading capacity. Thus, G3 DGL were selected as the nanoprobe scaffold. Maleimide (Mal) and N-hydroxysuccinimidyl (NHS) esterfunctionalized polyethylene glycol (PEG) served both as a linker and as a stealth coating. The synthesis of $\mathrm{CD}$ has been previously report$\mathrm{ed}^{14} \cdot 2$-(4-Isothiocyanatobenzyl)-diethylenetriaminepentaacetic acid a

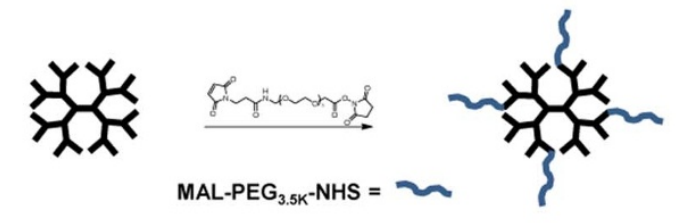

G3 DGL $\left(\mathrm{NH}_{2}\right)_{123}$

DGL-PEG (DP)

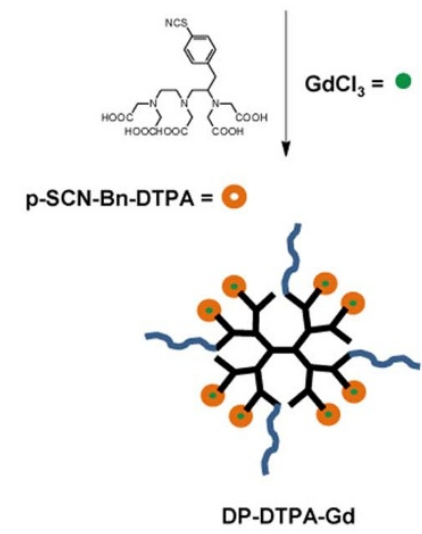

p-SCN-Bn-DTPA =

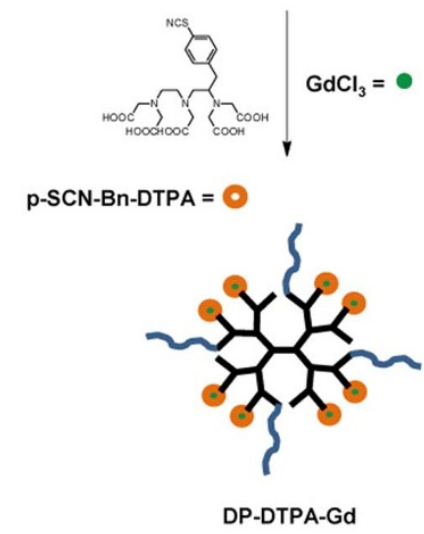

DP-DTPA-Gd

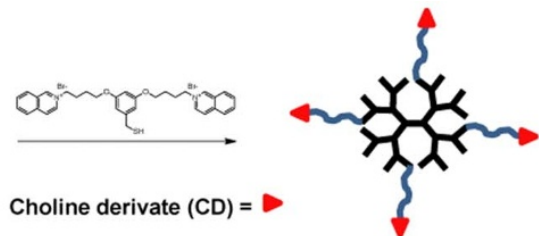

DGL-PEG-CD (DPC)
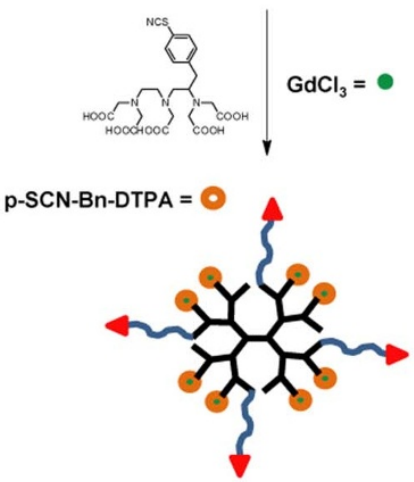

DPC-DTPA-Gd

b

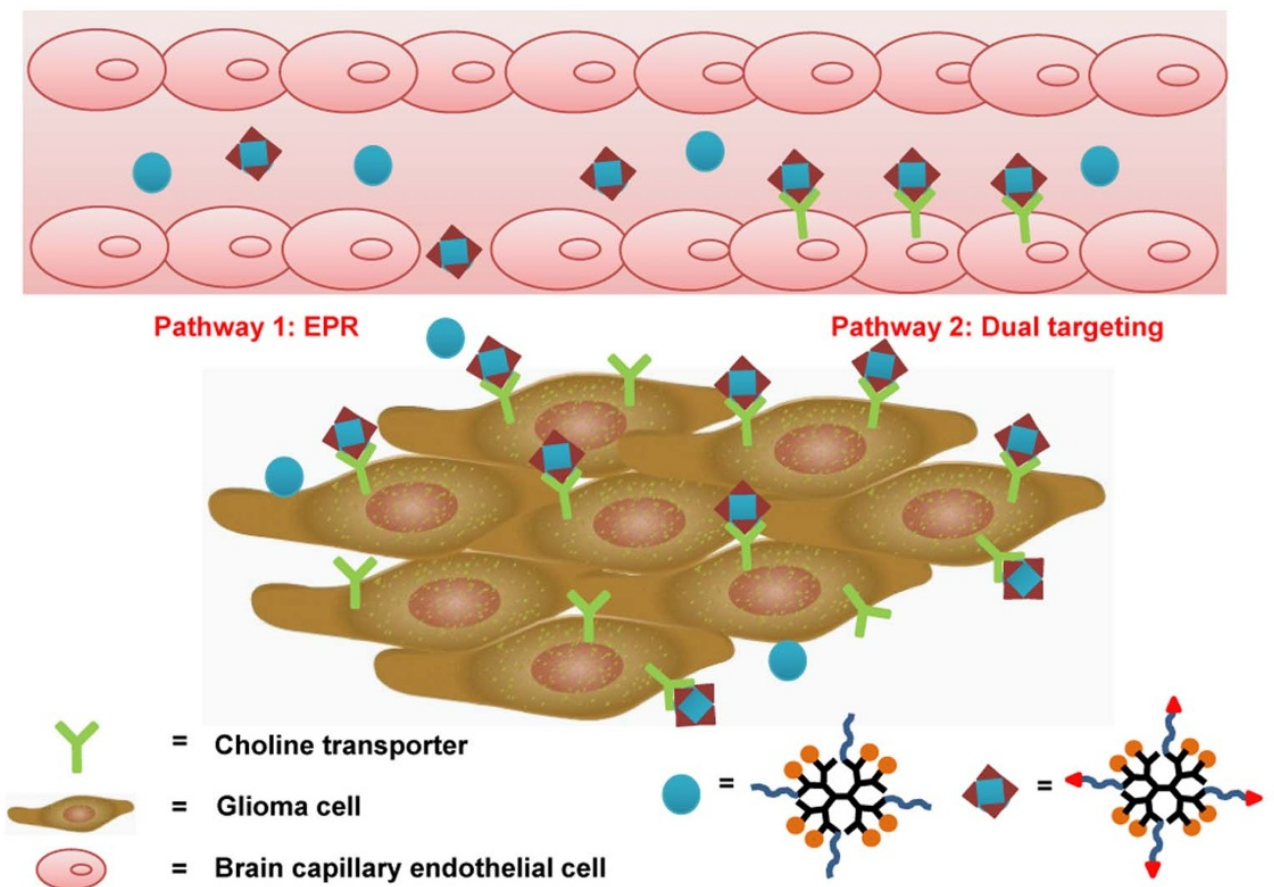

Figure $1 \mid$ Synthetic route for the nanoprobes and their targeting strategies. (a) DGL and CD were linked using MAL-PEG $3.5 \mathrm{~K}-\mathrm{NHS}$. Paramagnetic $\mathrm{Gd}^{3+}$ ions were conjugated to DP or DPC using p-SCN-Bn-DTPA. (b) The DP-DTPA-Gd nanoprobe accumulated in glioma via the EPR effect, whereas the DPC-DTPA-Gd nanoprobes could also enter the glioma via choline transporters, which are on brain capillary endothelial cells and glioma cells. 
(p-SCN-Bn-DTPA) is an activated chelating agent that can be conjugated to the surface of a scaffold under mild conditions. The targeting nanoprobe DPC-DTPA-Gd and control nanoprobe DPDTPA-Gd were synthesized as illustrated in Figure 1a. Briefly, MAL-PEG ${ }_{3.5 \mathrm{~K}}$-NHS treated with DGL produced DGL-PEG (DP), which was further reacted with the CD to generate DGL-PEG-CD (DPC). p-SCN-Bn-DTPA was conjugated to DP or DPC and then complexed with $\mathrm{Gd}^{3+}$ ions to yield DP-DTPA-Gd and DPC-DTPA$\mathrm{Gd}$, respectively. PEG modification rescued the nanoprobes from the reticuloendothelial system (RES), and DTPA conjugation furthered masked the surface charge of DGL. Thus, DP-DTPA-Gd, with a prolonged circulating half-life, accumulated in the glioma via an EPR effect. CD modification was expected to demonstrate the abovementioned proof of concept: ChT-mediated BBB permeation with subsequent ChT mediated internalization of the nanoprobe into gliomas (Fig. 1b).

Nanoprobe characterisation. In the ${ }^{1} \mathrm{H}$ NMR spectra, the peaks between 1 and $2 \mathrm{ppm}$ represent $\mathrm{H}$ protons of the $3 \mathrm{CH}_{2}$ in lysine units. Peaks near $3.6 \mathrm{ppm}$ indicate repeat units in PEG. The protons in the $\mathrm{CD}$ isoquinoline moieties range from 7.8 to $8.6 \mathrm{ppm}$, whereas the benzyl group in p-SCN-Bn-DTPA is at $7.2 \mathrm{ppm}$. The calculated DGL: PEG:CD : DTPA molar ratio was $1: 10.23: 5.37: 72.26$ (Fig. 2a). The amount of Gd chelated was determined using inductively coupled plasma atomic emission spectroscopy (ICP-AES). The mass percentages for $\mathrm{Gd}^{3+}$ in DP-DTPA-Gd and DPC-DTPA-Gd were $13.62 \%$ and $11.07 \%$, respectively. Atomic force microscopy (AFM) showed a narrow size distribution for DPC-DTPA-Gd $(18.0 \mathrm{~nm}$,
Fig. 2b) and DP-DTPA-Gd (16.2 nm). The relaxivity $\left(\mathrm{r}_{1}\right)$ of the nanoprobes was calculated as the slope of the plot of $1 / \mathrm{T}_{1}$ as a function of the gadolinium concentration (Fig. 2c). The $\mathrm{T}_{1}$ relaxation times were determined using a Bruker Biospec $4.7 \mathrm{~T} / 30$ $\mathrm{cm}$ scanner. The calculated $\mathrm{r}_{1}$ values for DP-DTPA-Gd and DPCDTPA-Gd were 8.78 and $12.96 \mathrm{mM}^{-1} \mathrm{~s}^{-1}$ per $\mathrm{Gd}$, whereas the calculated $r_{1}$ value for DTPA-Gd was only $4.57 \mathrm{mM}^{-1} \mathrm{~s}^{-1}$. The $\mathrm{r}_{1}$ value for the nanoprobe with a different CD density (DPC*-DTPAGd, 2 CD per DGL) was $10.21 \mathrm{mM}^{-1} \mathrm{~s}^{-1}$ per Gd. As reported by Yan et $a .^{17}$, the nanoprobe $\mathrm{r}_{1}$ values ranged from $6.9-7.4 \mathrm{mM}^{-1} \mathrm{~s}^{-1}$. Peptide ligand conjugation did not affect this parameter, whereas the bis-quaternary ammonium nature of the CD may affect either the water exchange rate or the rotational correlation time. The precise mechanism will be investigated in the future.

ChT affinity assay and cellular uptake of the scaffolds. The glioma ChT affinity for the scaffolds was evaluated as the inhibition efficiency of $\left[{ }^{3} \mathrm{H}\right]$-choline chloride uptake by U87 MG cells (Fig. 3a). The uptake of the CD group $(23.05 \pm 0.72 \%)$ was lower than for the choline chloride group $(51.32 \pm 4.65 \%)$, which indicates that CD had a higher glioma ChT affinity than choline chloride. The nanoprobe treated with DPC exhibited a slightly greater $\left[{ }^{3} \mathrm{H}\right]$-choline chloride uptake $(37.89 \pm 10.75 \%)$, whereas the DP group showed little inhibition $(95.92 \pm 2.91 \%)$. Glioma ChT affinity for the CD remained after DP conjugation. To confirm whether the CD could mediate scaffold accumulation in gliomas, cells were incubated with BODIPY-labelled scaffolds for $30 \mathrm{~min}$ and were then examined using fluorescence microscopy (Fig. 3b). Cellular uptake of DPC was

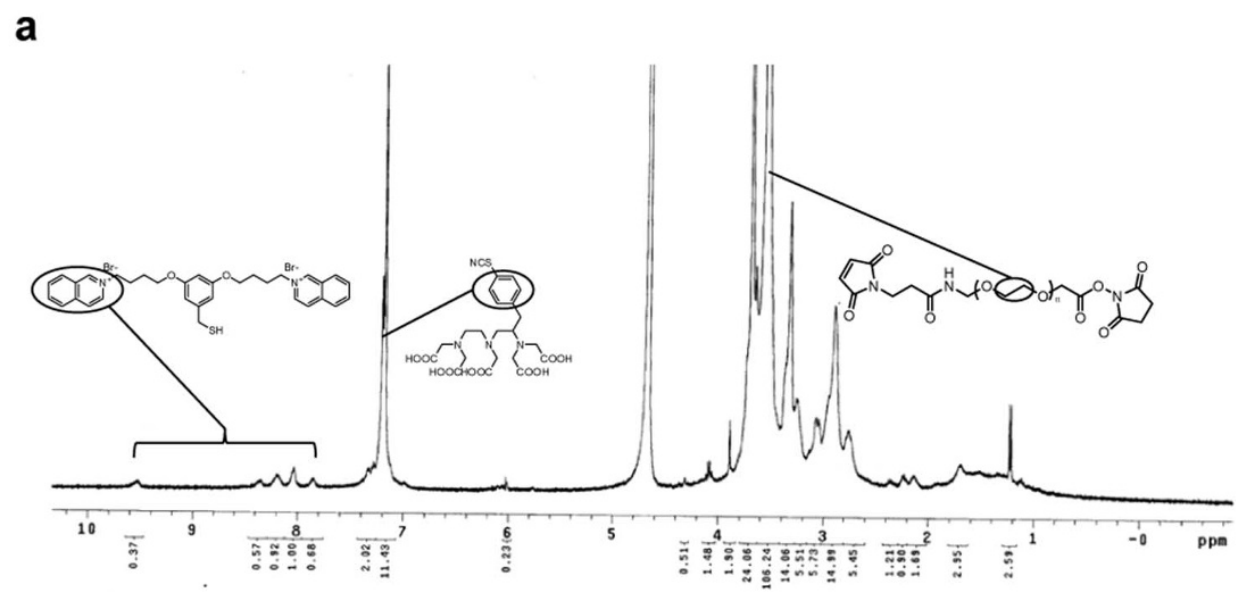

b

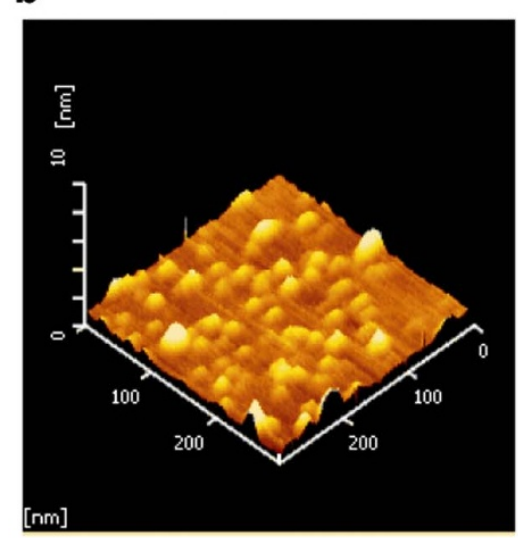

c

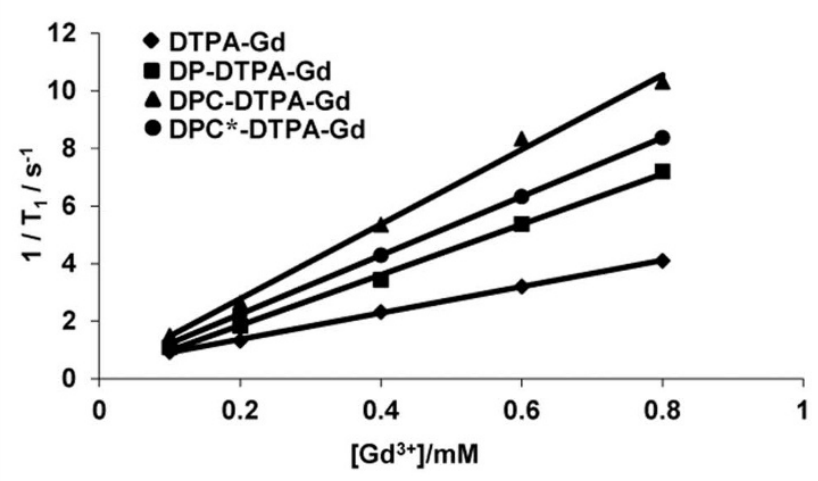

Figure $2 \mid$ Nanoprobe characterisation. (a) ${ }^{1} \mathrm{H}$ NMR spectra for DPC-DTPA. (b) An AFM image of the DPC-DTPA-Gd nanoprobe. (c) Relaxivity ( $\mathrm{r}_{1}$ )

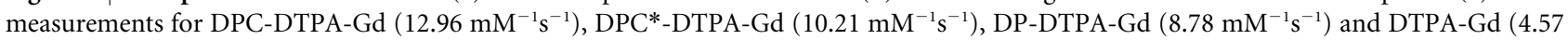
$\left.\mathrm{mM}^{-1} \mathrm{~s}^{-1}\right)$. 
a

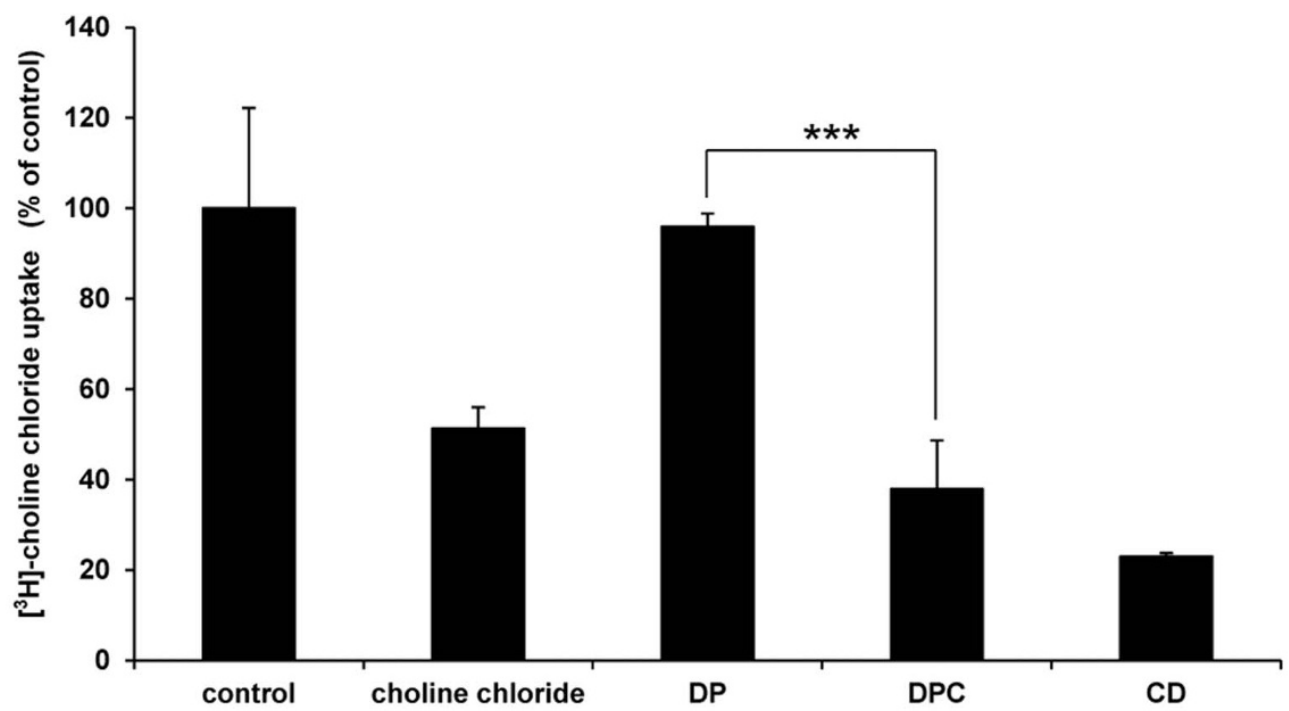

b
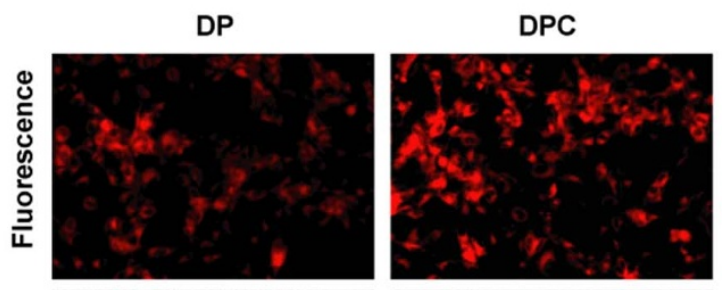

DPC+choline chloride

DPC $4{ }^{\circ} \mathrm{C}$
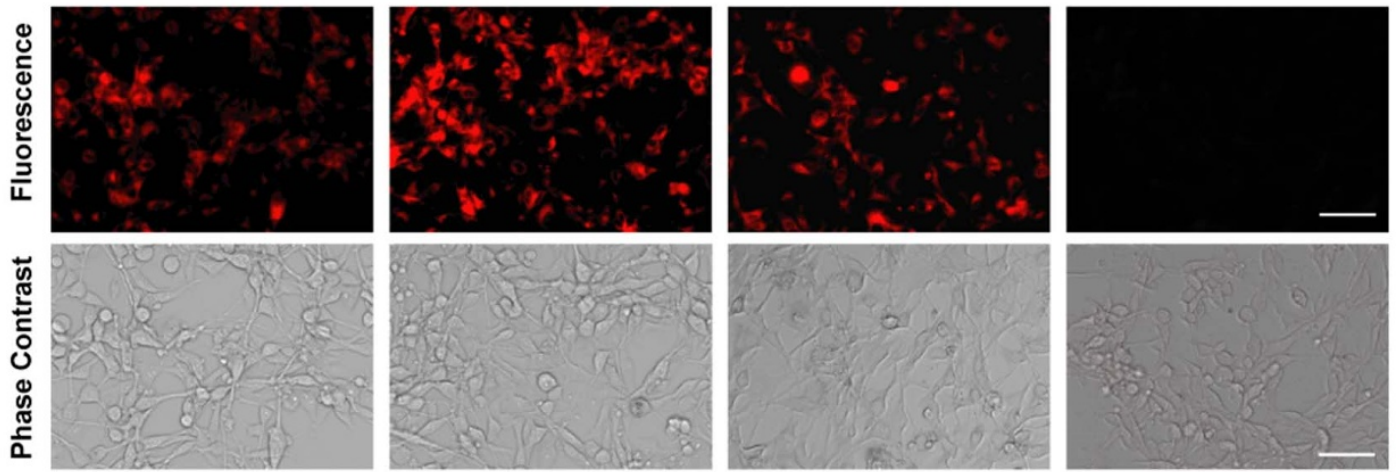

Figure 3 ChT affinity assay and cellular uptake of scaffolds. (a) U87 MG uptake of $10 \mathrm{nM}\left[{ }^{3} \mathrm{H}\right]$-choline chloride was measured after the cells were incubated for $10 \mathrm{~min}$ with $\mathrm{NaCl}$ at $\mathrm{pH} 7.4$ either without (control) or with $10 \mu \mathrm{M}$ choline chloride, DP, DPC or CD. The data are expressed as the mean \pm S.D. $(\mathrm{n}=4), \mathrm{p}=0.026$, unpaired t-test. (b) Cellular uptake of BODIPY-labelled scaffolds after a 30 -min incubation at $37^{\circ} \mathrm{C}$ or $4^{\circ} \mathrm{C}$. Scale bars: $100 \mu \mathrm{m}$.

a

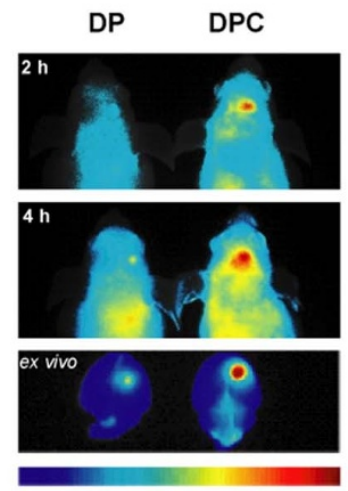

b
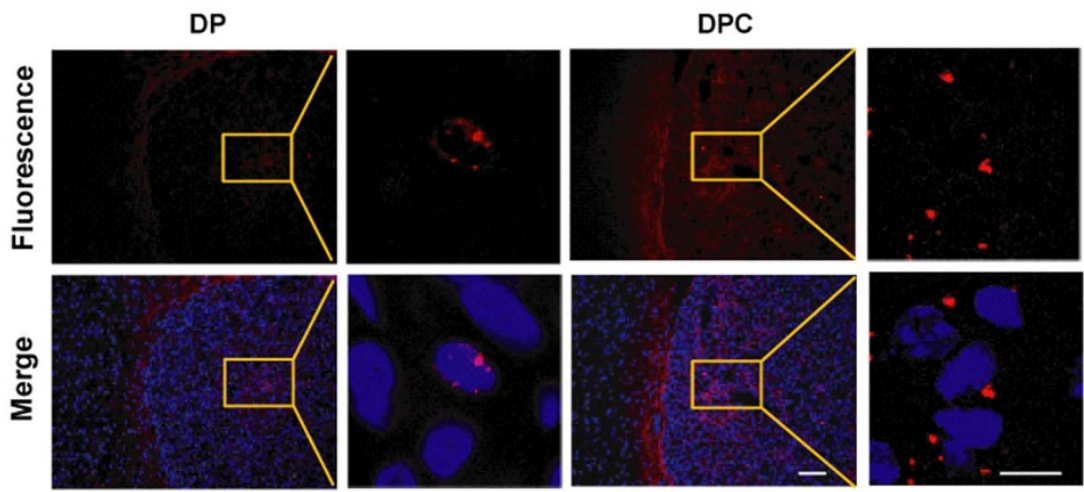

Figure $4 \mid$ In vivo and ex vivo images for U87 MG-bearing nude mice administered scaffolds. (a) Fluorescence images were taken 2 and 4 h after i.v. injection of BODIPY-labelled DP and DPC. Four hours post-injection, ex vivo images of the brain were generated. (b) Frozen sections were examined using fluorescence and confocal microscopy. Red: BODIPY-labelled scaffolds. Blue: DAPI-labelled nucleus. Scale bars for fluorescence microscopy: $100 \mu \mathrm{m}$; for confocal microscopy: $5 \mu \mathrm{m}$. 
a
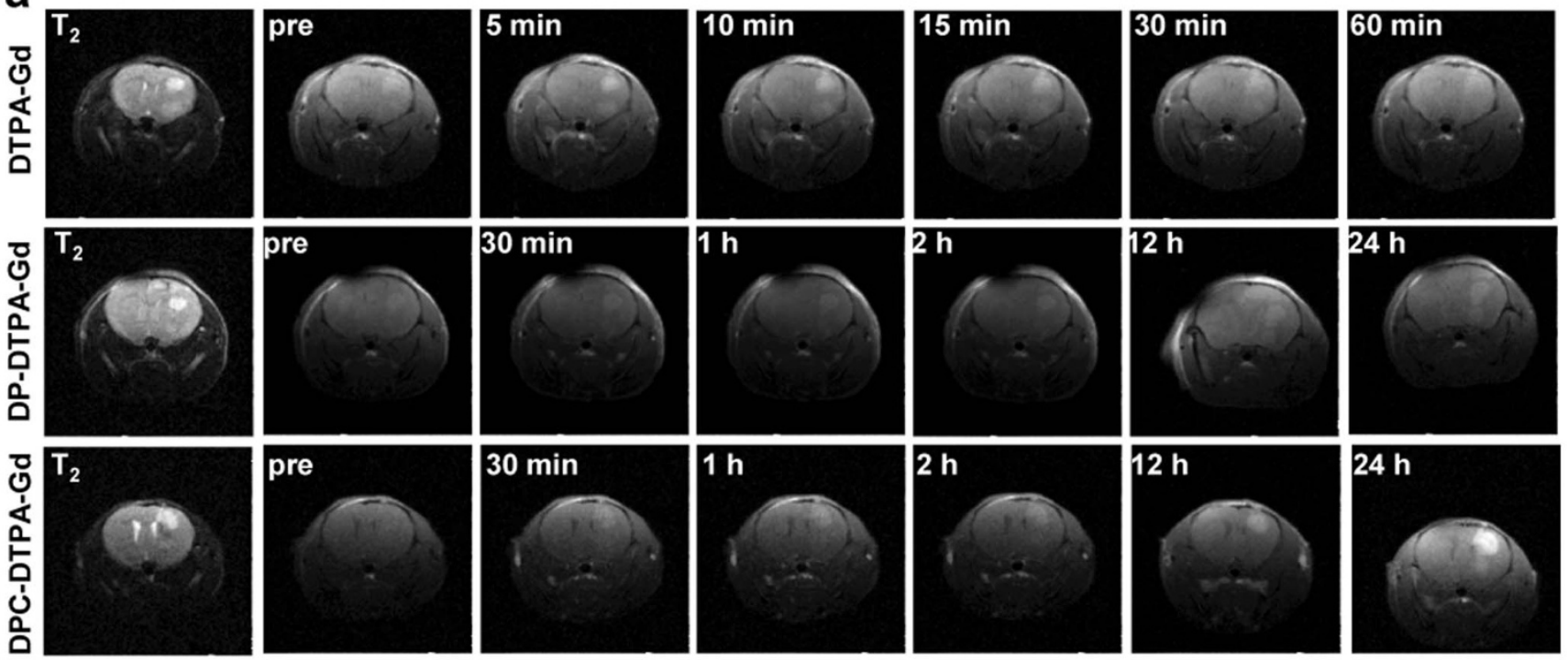

b
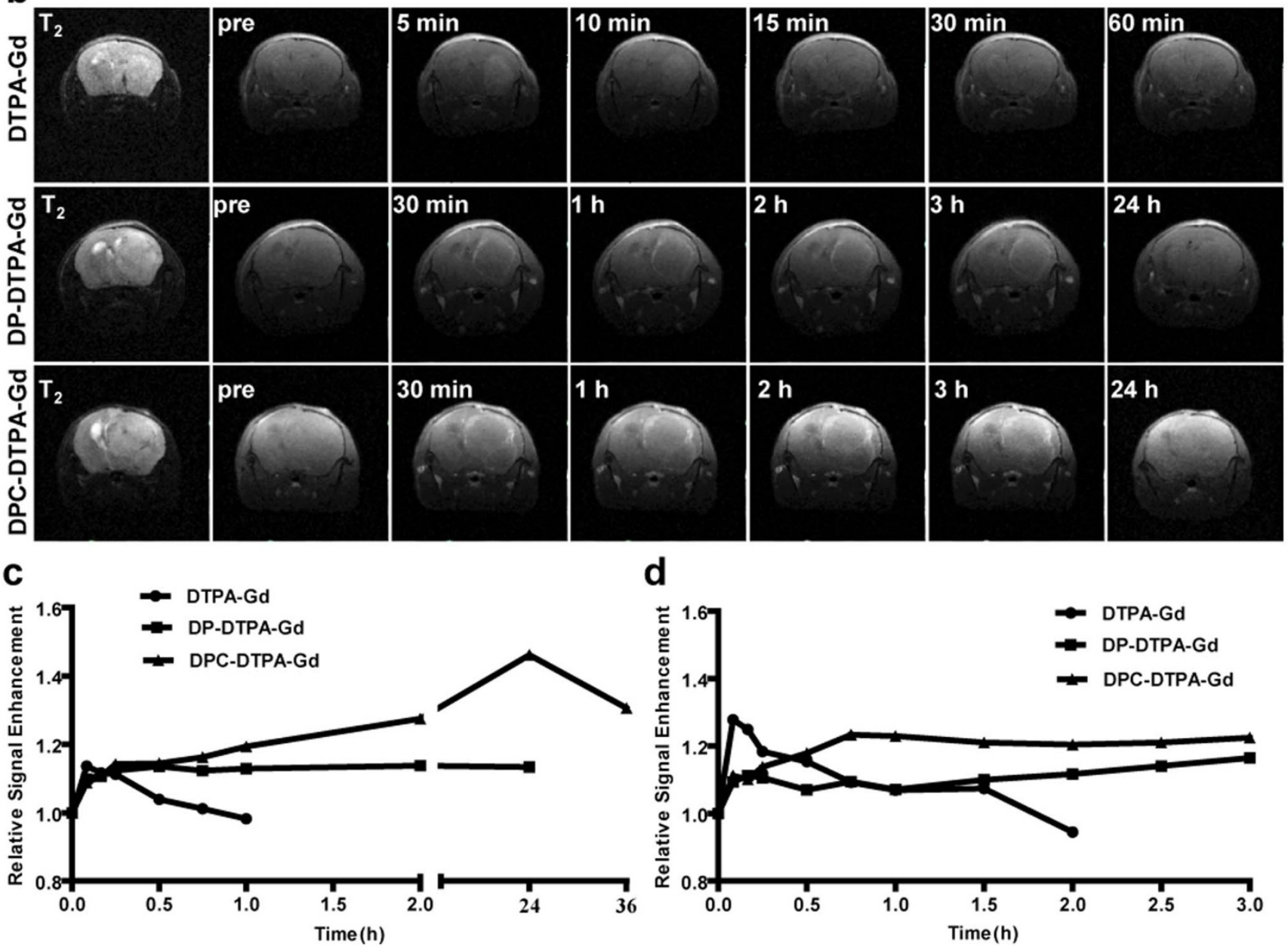

Figure 5 Dynamic MRI and quantification of the signal enhancement in glioma-bearing nude mice pre-contrast and at various time points after i.v. injection of different probes. (a) Nude mice bearing small-volume glioma were administered DTPA-Gd (top row), DP-DTPA-Gd (second row) or DPC-DTPA-Gd (third row). (b) Nude mice bearing large-volume glioma were administered DTPA-Gd (top row), DP-DTPA-Gd (second row) or DPC-DTPA-Gd (third row). (c), (d) Quantitative analysis results for the MR images. The average MR signal intensity was measured for each tumor, and the relative signal enhancement was then calculated as the quotient of the intensity at various time points for the pre- and post-contrast images. 
significantly higher than that of DP and was inhibited by excessive choline chloride or by a decrease in temperature to $4^{\circ} \mathrm{C}$.

In vivo evaluation of scaffold-targeting efficacy. In vivo fluorescence imaging was performed to trace BODIPY-labelled scaffold distribution in U87 MG-bearing nude mice 2 and $4 \mathrm{~h}$ after intravenous (i.v.) administration (Fig. 4a). DPC showed a significant accumulation in the tumor region $4 \mathrm{~h}$ after injection, whereas the DP-treated mice produced no obvious signal. Ex vivo fluorescence imaging generated an even higher signal contrast between tumors and normal brains in DPC-treated mice. The tumor sections were examined using fluorescence and confocal microscopy (Fig. 4b). Consistent with the in vivo imaging results, tumor accumulation of DPC was significantly greater than that of DP. These results demonstrate the potential glioma-targeting efficiency of the DPC scaffold.

Dynamic MRI and quantification of signal enhancement. To examine whether DPC-DTPA-Gd could be used to effectively identify gliomas in vivo, dynamic MRI was performed pre-contrast and at various time points after i.v. injection of DP-DTPA-Gd, DPCDTPA-Gd or DTPA-Gd $(0.05 \mathrm{mmol} \mathrm{Gd} / \mathrm{kg})$. Prior to $\mathrm{T}_{1}$-weighted imaging, $\mathrm{T}_{2}$ imaging was performed to confirm the volume and localization of the glioma. In the small-volume glioma mice, DTPAGd administration produced the greatest contrast enhancement $(113.6 \%)$ at 5 min after i.v. injection. The signal enhancement then decreased through rapid renal clearance of DTPA-Gd from the blood pool. DP-DTPA-Gd administration produced a slight contrast enhancement during the first 2 hours and then plateaued for $24 \mathrm{~h}$. DPC-DTPA-Gd administration significantly increased the contrast enhancement by $24 \mathrm{~h}$, and the image clearly delineated the tumor boundary. Quantitative analysis (146.1\%) further demonstrated the targeting efficacy of DPC-DTPA-Gd (Fig. 5a, c). In large-volume glioma mice, the signal enhancement trend after DTPA-Gd administration was similar to the former observation. Both nanoprobes induced a ring-enhancing lesion, which is a classic enhancement pattern that has been demonstrated in high-grade gliomas ${ }^{15}$. A thicker ring lesion was observed upon DPC-DTPA-Gd injection.

\section{Discussion}

Schlageter et al. have systemically investigated microvessel organisation and structure in experimental brain tumors and identified the following three distinct populations of brain tumor microvessels: (1) continuous nonfenestrated microvessels, through which drugs predominantly enter by passive diffusion; (2) continuous fenestrated microvessels, which have selectively increased permeability over the normal brain; and (3) microvessels with endothelial gaps, which exhibit increased permeability to water-soluble compounds ${ }^{16}$. We used an in situ implantation method to establish our experimental model. This invasive method disrupted the normal BBB. When gliomas develop, the brain vasculature is substituted by tumor neovasculature to support glioma growth. This is the fundament of EPR effect that supports our nanoparticle probe design. However, glioma cells near the boundary of tumor region could infiltrate into the normal brain and use the existing brain vasculature with a largely intact BBB. In this study, the CD-modified nanoprobe was designed based on the above-mentioned pathological characteristics of gliomas to achieve the possibility of imaging this part of glioma.

One primary characteristic is the blood-brain tumor barrier (BBTB). Brain tumor vasculature (BTV) retains certain features of the BBB, such as tight interendothelial junctions and transendothelial channels ${ }^{17}$. The physiological upper limit of the BBTB pore size is much smaller than in other solid tumors ${ }^{8}$. Nanoprobes with smaller sizes would more efficiently permeate the glioma region. However, nanoparticles smaller than 5 to $6 \mathrm{~nm}$ that weigh less than 30 to $40 \mathrm{kD}$ are efficiently filtered by the kidneys ${ }^{18}$. Only nanoparticles at an appropriate size have longer circulating half-lives and more efficient glioma accumulation. In this study, the selected dendrimer G3 DGL was $7 \mathrm{~nm}$. The hydrodynamic diameter of the resulting nanoprobes was $30.0 \pm 3.5 \mathrm{~nm}$ for DP-DTPA-Gd and $32.1 \pm 0.2 \mathrm{~nm}$ for DPCDTPA-Gd, respectively. The signal enhancement for both nanoprobes was maintained for more than $24 \mathrm{~h}$ in the small-volume glioma, which indicates a long blood half-life (Fig. 5c). An additional advantage from nanoprobes is the high relaxivity $r_{1}$ value (Fig. $2 c$ ). Large-size nanoprobes can significantly lengthen the rotational correlation for Gd-chelates (longer than nanoseconds) to increase relaxivity $^{19}$. Modern MRI instruments operate at high magnetic fields, where contrast agents have low relaxivity. Typical relaxivities for such compounds are approximately $4-5 \mathrm{mM}^{-1} \mathrm{~s}^{-1}\left(4.57 \mathrm{mM}^{-1} \mathrm{~s}^{-1}\right.$ as calculated in our experiments), whereas the theoretical values could be increased to greater than $100 \mathrm{mM}^{-1} \mathrm{~s}^{-1}$. According to the Bloembergen-Solomon-Morgan (BSM) theory, rotational correlation time is a critical factor governing relaxivity. If molecular tumbling is too fast, relaxivity enhancement is limited. To slow the rotational motion, gadolinium complexes can be covalently conjugated to nanosize carriers with different sizes and shapes (dendrimers, polysaccharides, micelles and liposomes). Thus, nanoprobes are more efficient than small molecular contrast agents.

Although PEG and DTPA conjugation could rescue the nanoprobes from the RES by masking the surface charge on DGL, the negative charge further prevented tumor internalization of the nanoprobes $(-13.1 \pm 0.9 \mathrm{mV}$ for DP-DTPA-Gd). Signal enhancement for DP-DTPA-Gd reached a plateau $2 \mathrm{~h}$ after administration (Fig. 5c). Thus, active targeting should be used to increase cellular internalization.

An additional primary characteristic of gliomas is the $\mathrm{BBB}$ at the tumor margin. Although $\left({ }^{18} \mathrm{~F}\right)$-labelled choline has been demonstrated to be a novel glioma imaging tracer, successful conjugation to nanoprobes has been limited, likely because the quaternary ammonium group and free hydroxyl group are key requirements for maintaining ChT affinity ${ }^{20}$. We previously showed that a novel CD has higher BBB ChT affinity than choline chloride ${ }^{14}$. Here, $\left[{ }^{3} \mathrm{H}\right]-$ choline chloride uptake inhibition demonstrated that the $\mathrm{CD}$ also has higher glioma affinity compared with choline chloride (Fig. 2a). Thus, this CD with both higher BBB and ChT affinity was used as a dual targeting ligand that can be easily conjugated to the scaffold through a Michael reaction (Fig. 1a). Cellular uptake of BODIPYlabelled scaffolds provides direct evidence that the $\mathrm{CD}$ mediated not only ChT affinity but also cellular uptake of the scaffolds both in vitro and in vivo (Figs. 2b, 3). In Fig. $5 \mathrm{a}, \mathrm{T}_{1}$ weighted imaging $24 \mathrm{~h}$ post DPC-DTPA-Gd administration revealed a larger enhancing region than both $12 \mathrm{~h}$ post DPC-DTPA-Gd administration and $\mathrm{T}_{2}$ weighted images. In Fig. 5b, $\mathrm{T}_{1}$ weighted imaging after DPC-DTPA-Gd administration showed a thicker ring-enhancing lesion than $\mathrm{T}_{1}$ weighted imaging after DP-DTPA-Gd administration. This region uptakes high levels of fluorocholine ${ }^{21}$. The malignancy contributed to high DPC-DTPA-Gd uptake, which produced a thicker ring-enhancing lesion. However, hyperosmosis or necrosis inside the tumor region prevented efficient accumulation of the nanosize probes in a deeper tumor region. Irrespective of the tumor volume, both of these results indicated the superior dual targeting of DPC-DTPA-Gd.

In conclusion, we developed a novel BBB ChT and glioma ChT dual-targeting nanoprobe for precise glioma detection and tumor boundary delineation. The targeting strategy employed here may provide a new method for glioma diagnosis. In addition, the high tumor-to-normal ratio observed under in vivo and ex vivo fluorescence imaging indicates that the targeting scaffold is promising for fluorescence-guided brain tumor resection during surgery.

\section{Methods}

Materials. DGL with 123 primary amino groups (generation 3) was purchased from Colcom (Montpellier Cedex, France, France). a-Maleimidyl-u- $N$ -

hydroxysuccinimidyl polyethylene glycol (NHS-PEG-MAL, MW 3500) was obtained from Jenkem Technology (Beijing, China). (p-SCN-Bn-DTPA) was purchased from 
Macrocyclics (Dallas, TX, USA). Gadopentetate dimeglumine injection (DTPA-Gd) was purchased from Beijing Beilu Pharmaceutical (Beijing, China). 6-((4,4-Difluoro5-(2-pyrrolyl)-4-bora-3a,4a-diaza-s-indacene-3-yl)styryloxy) acetyl) aminohexanoic acid, succinimidyl ester (BODIPY) was purchased from Molecular Probes (Eugene, OR, USA). $\left[{ }^{3} \mathrm{H}\right]$-choline chloride $(80.6 \mathrm{Ci} / \mathrm{mmol})$ was purchased from PerkinElmer (USA). Unless specified otherwise, all other chemicals were purchased from Sigma-Aldrich and were used without further purification.

Synthesis and characterization of nanoprobes. DGL reacted with $\mathrm{NHS}-\mathrm{PEG}_{3.5 \mathrm{~K}^{-}}$ MAL at a $1: 10$ molar ratio in PBS 7.4 for $0.5 \mathrm{~h}$ at room temperature (RT). The primary amino groups on the surface of DGL specifically reacted with the NHS groups in the bifunctional PEG derivative. The resulting conjugate, DGL-PEG, was purified by ultrafiltration through a membrane (cutoff $=5 \mathrm{kDa}$ ). DGL-PEG then reacted with the $C D$ at a $1: 10$ molar ratio in PBS 7.4 for $2 \mathrm{~h}$ at RT. The MAL groups in DGL-PEG specifically reacted with the $\mathrm{CD}$ thiol groups. The resulting conjugate, DPC, was purified by ultrafiltration through a membrane (cutoff $=10 \mathrm{kDa}$ ). The scaffolds generated, DP and DPC, were lyophilized. The DP and DPC scaffolds then reacted with p-SCN-Bn-DTPA (DGL: p-SCN-Bn-DTPA $=1: 246$, mol:mol) in HEPES 8.5 for $24 \mathrm{~h}$ at RT. The conjugate produced, DP-DTPA or DPC-DTPA, was purified by ultrafiltration through a membrane (cutoff $=10 \mathrm{kDa}$ ) and was redissolved in $0.1 \mathrm{M} \mathrm{Na}$-acetate buffer (pH 6.0). Gadolinium(III) chloride hexahydrate (DGL: $\left.\mathrm{Gd}^{3+}=1: 246\right)$ was added and allowed to react for an additional $24 \mathrm{~h}$ at $4{ }^{\circ} \mathrm{C}$. The characteristics of DPC-DTPA were analyzed using NMR spectroscopy. The number of Gd particles per DGL was measured using ICP-AES (Varian, Palo Alto, CA, USA). The nanoprobe particle size and zeta potential were determined using dynamic light scattering and a NicompTM 380 ZLS zeta-potential/particle sizer (PSS Nicomp Particle Size System, USA). The nanoprobe morphologies were examined using AFM. The longitudinal relaxation-time measurements were performed using a Bruker Biospec $4.7 \mathrm{~T} / 30 \mathrm{~cm}$ scanner. Relaxivity $\mathrm{r}_{1}$ was determined through the fitting of the plot of $\mathrm{R}_{1}$ as a function of $\left[\mathrm{Gd}^{3+}\right]$ using a linear function.

Inhibition of $\left[{ }^{3} \mathbf{H}\right]$-choline chloride uptake. U87 MG glioma cells were expanded and maintained in Dulbecco's modified Eagle medium (DMEM) supplemented with $10 \% \mathrm{FBS}, 100 \mathrm{U} / \mathrm{mL}$ penicillin and $100 \mu \mathrm{g} / \mathrm{mL}$ streptomycin; the cells were then cultured at $37^{\circ} \mathrm{C}$ under a humidified atmosphere that contained $5 \% \mathrm{CO}_{2}$. U87 MG cells were seeded at $5 \times 10^{4}$ cells/well in 24-well plates (Corning-Coaster, Tokyo, Japan). The cells were incubated for $48 \mathrm{~h}$ and then observed under the microscope for confluency and morphology. U87 MG uptake of $10 \mathrm{nM}\left[{ }^{3} \mathrm{H}\right]$-choline chloride (dissolved in $0.25 \mathrm{~mL}$ of Hanks' buffer) was measured the cells were incubation for 10 min either without (control) or with $10 \mu \mathrm{M}$ choline chloride, DP, DPC or CD. The unlabelled choline chloride served as a positive control. Uptake was terminated through aspiration of the incubation buffer and three washings of the cells ice-cold Hanks' buffer. A $2 \mathrm{~N} \mathrm{NaOH}$ solution $(0.2 \mathrm{~mL})$ was added to each well to lyse the cells and was subsequently neutralized with $4 \mathrm{~N} \mathrm{HCl}(0.1 \mathrm{~mL})$. To determine the radioactivity, a scintillant liquid $(2 \mathrm{~mL})$ was added to the solubilized cell solution $(0.1 \mathrm{~mL})$, and the radioactivity was measured using a liquid scintillation counter (LS 6000SE, Beckman, USA).

Cellular uptake of BODIPY-labelled scaffolds. To synthesise BODIPY-labelled scaffolds, DGL was dissolved in a $100 \mathrm{mM} \mathrm{NaHCO}_{3}$ solution followed by the addition of BODIPY (5 equiv.) in DMSO; the reaction mixture was then incubated for $1 \mathrm{~h}$ at RT and purified via ultrafiltration using a $3 \mathrm{kDa}$ molecular-weight cutoff membrane to remove the unreacted BODIPY. The BODIPY-labelled DGL was used to synthesize different BODIPY-labelled scaffolds as previously described. U87 MG cells were seeded and incubated as described previously. After the confluency and morphology were observed, the medium was removed, and the cells were treated with BODIPYlabelled DP or DPC at $15 \mu \mathrm{g} \mathrm{DGL} /$ well in a serum-free medium for $30 \mathrm{~min}$ at $37^{\circ} \mathrm{C}$. Excessive choline chloride was added as an inhibitor. BODIPY-labelled DPC uptake was also performed at $4^{\circ} \mathrm{C}$.

In vivo fluorescence imaging. Male nude mice (4-6 weeks old) with body weights of 18-20 g were supplied by the Department of Experimental Animals, Fudan University and were maintained under standard housing conditions. All animal experiments were performed in accordance with guidelines evaluated and approved by the ethics committee of Fudan University. To prepare the U87 MG glioma model, $2 \times 10^{5} \mathrm{U} 87$ MG cells in $5 \mu \mathrm{l}$ of PBS were injected into the right striatum $(1.8 \mathrm{~mm}$ lateral to the bregma and $3 \mathrm{~mm}$ deep) using a stereotactic fixation device with a mouse adaptor. Twenty-one days after implant, the BODIPY-labelled DP and DPC were injected into the tail vein of model mice at $150 \mu \mathrm{g}$ DGL/mouse, respectively. At 2 and $4 \mathrm{~h}$, the model mice were anesthetized and visualized using a Cambridge Research \& Instrumentation (CRi) in vivo imaging system (CRi, MA, USA). The mice were then sacrificed, and the brains were removed and fixed in $4 \%$ paraformaldehyde. Ex vivo imaging was also performed using a CRi in vivo imaging system. Thereafter, the brains were frozen in an OCT-embedding medium (Sakura, Torrance, CA, USA) at $-80^{\circ} \mathrm{C}$. The frozen sections were $20 \mu \mathrm{m}$ thick; they were prepared with a cryotome cryostat (Leica, CM 1900, Wetzlar, Germany) and then stained with $300 \mathrm{nM}$ DAPI for $10 \mathrm{~min}$ at RT. After being washed twice with PBS (pH 7.4), the sections were immediately examined under a confocal microscope.

MRI. U87 MG-bearing nude mice were scanned using a Bruker Biospec $4.7 \mathrm{~T} / 30 \mathrm{~cm}$ scanner. The gradients used in the magnet were a diameter of $12 \mathrm{~cm}$ at $25 \mathrm{G} / \mathrm{cm}$. Imaging was performed using a custom-built $50 \mathrm{~mm}$ diameter send-receive birdcage volume coil. The $\mathrm{T}_{1}$-weighted images were produced using the following parameters: slice thickness $=1.00 \mathrm{~mm}, \mathrm{FOV}$ (field of view) $=2 \times 2 \mathrm{~cm}^{2}, \mathrm{TE}=11 \mathrm{~ms}, \mathrm{TR}=300$ $\mathrm{ms}, \mathrm{Na}$ (number of averages) $=8$ and matrix size $=128 \times 128$. The scans were completed at different time points after the i.v. administration of DTPA-Gd, DP-DTPA-Gd and DPC-DTPA-Gd at $0.05 \mathrm{mmol} \mathrm{Gd} / \mathrm{kg}$. The $\mathrm{T}_{2}$-weighted images were also generated through monitoring of the glioma volume. To quantitatively analyze the MR images, we measured the average MR signal intensities (SI) in the regions of interest (ROI) surrounding the tumors. Relative signal enhancement was calculated as the SI quotient for the pre- and post-contrast images.

1. Huse, J. T. \& Holland, E. C. Targeting brain cancer: advances in the molecular pathology of malignant glioma and medulloblastoma. Nat Rev Cancer 10, 319-331 (2010).

2. Wen, P. Y. \& Kesari, S. Malignant gliomas in adults. N Engl J Med 359, 492-507 (2008).

3. Bellin, M. F. MR contrast agents, the old and the new. Eur J Radiol 60, 314-323 (2006).

4. Scott, J. N., Brasher, P. M., Sevick, R. J., Rewcastle, N. B. \& Forsyth, P. A. How often are nonenhancing supratentorial gliomas malignant? A population study. Neurology 59, 947-949 (2002)

5. Caravan, P., Ellison, J. J., McMurry, T. J. \& Lauffer, R. B. Gadolinium(III) Chelates as MRI Contrast Agents: Structure, Dynamics, and Applications. Chem Rev 99, 2293-2352 (1999).

6. Han, L. et al. Peptide-conjugated polyamidoamine dendrimer as a nanoscale tumor-targeted T1 magnetic resonance imaging contrast agent. Biomaterials 32, 2989-2998 (2011).

7. Huang, R. et al. Chlorotoxin-modified macromolecular contrast agent for MRI tumor diagnosis. Biomaterials 32, 5177-5186 (2011).

8. Sarin, H. et al. Effective transvascular delivery of nanoparticles across the blood-brain tumor barrier into malignant glioma cells. J Transl Med 6, 80 (2008).

9. Michel, V., Yuan, Z., Ramsubir, S. \& Bakovic, M. Choline transport for phospholipid synthesis. Exp Biol Med (Maywood) 231, 490-504 (2006)

10. Machova, E. et al. Detection of choline transporter-like 1 protein CTL1 in neuroblastoma $\times$ glioma cells and in the CNS, and its role in choline uptake. J Neurochem 110, 1297-1309 (2009).

11. Wang, T. et al. Choline transporters in human lung adenocarcinoma: expression and functional implications. Acta Biochim Biophys Sin (Shanghai) 39, 668-674 (2007).

12. Allen, D. D. \& Lockman, P. R. The blood-brain barrier choline transporter as a brain drug delivery vector. Life Sci 73, 1609-1615 (2003).

13. DeGrado, T. R. et al. Synthesis and evaluation of (18)F-labeled choline analogs as oncologic PET tracers. J Nucl Med 42, 1805-1814 (2001).

14. Li, J. et al. Choline-derivate-modified nanoparticles for brain-targeting gene delivery. Adv Mater 23, 4516-4520 (2011).

15. Lipsitz, D. et al. Glioblastoma multiforme: clinical findings, magnetic resonance imaging, and pathology in five dogs. Vet Pathol 40, 659-669 (2003).

16. Schlageter, K. E., Molnar, P., Lapin, G. D. \& Groothuis, D. R. Microvessel organization and structure in experimental brain tumors: microvessel populations with distinctive structural and functional properties. Microvasc Res 58, 312-328 (1999)

17. Yan, H. et al. Two-order targeted brain tumor imaging by using an optical/ paramagnetic nanoprobe across the blood brain barrier. ACS Nano 6, 410-420 (2012).

18. Choi, H. S. et al. Renal clearance of quantum dots. Nat Biotechnol 25, 1165-1170 (2007).

19. Liu, Y. \& Zhang, N. Gadolinium loaded nanoparticles in theranostic magnetic resonance imaging. Biomaterials 33, 5363-5375 (2012).

20. Geldenhuys, W. J. et al. 3D-QSAR study of bis-azaaromatic quaternary ammonium analogs at the blood-brain barrier choline transporter. Bioorg Med Chem 13, 4253-4261 (2005).

21. Kwee, S. A., Ko, J. P., Jiang, C. S., Watters, M. R. \& Coel, M. N. Solitary brain lesions enhancing at MR imaging: evaluation with fluorine 18 fluorocholine PET. Radiology 244, 557-565 (2007).

\section{Acknowledgements}

This work was supported by a grant from the National Natural Science Foundation of China (30973652), the National Natural Science Foundation of China (81172993) and the "Key new drug creation program" 2009ZX09310-006. The authors thank Prof. Jianhua Zhu (School of Pharmacy, Fudan University) for the $\left[{ }^{3} \mathrm{H}\right]$-choline chloride testing work.

\section{Author contributions}

J.F.L. and J.C. conceived the experiment, designed the project and wrote the paper. J.F.L. and S.X.H. performed the experiments with the assistance of K.S., Y.L., S.A., Y.Y.K., Y.B.G. and H.J.M.; X.X.W. performed the MRI experiments.

\section{Additional information}

Competing financial interests: The authors declare no competing financial interests. 
License: This work is licensed under a Creative Commons

Attribution-NonCommercial-NoDerivs 3.0 Unported License. To view a copy of this

license, visit http://creativecommons.org/licenses/by-nc-nd/3.0/
How to cite this article: Li, J.F. et al. A choline derivate-modified nanoprobe for glioma diagnosis using MRI. Sci. Rep. 3, 1623; DOI:10.1038/srep01623 (2013). 